\title{
ESTRATÉGIAS PARA AUMENTO DE SENSIBILIDADE EM ESPECTROFOTOMETRIA UV-VIS
}

\author{
Fábio R. P. Rocha*
}

Instituto de Química, Universidade de São Paulo, CP 26077, 05513-970 São Paulo - SP

Leonardo S. G. Teixeira

Departamento de Engenharia e Arquitetura, Universidade Salvador, Av. Cardeal da Silva, 132, 40220-141 Salvador - BA

Recebido em 6/5/03; aceito em 19/2/04; publicado na web em 17/6/04

\begin{abstract}
STRATEGIES TO INCREASE SENSITIVITY IN UV-VIS SPECTROPHOTOMETRY. Spectrophotometry is one of the most widespread analytical techniques due to its simplicity, reliability, and low-cost instrumentation for both direct measurements and coupled to other techniques or processes such as chromatography, electrophoresis and flow analysis. However, the application is often limited by sensitivity. This article describes some advances that greatly improve the performance of spectrophotometric measurements, especially in order to increase sensitivity, including the employment of liquid-core waveguides and solid-phase spectrophotometry.
\end{abstract}

Keywords: UV-VIS spectrophotometry; long-pathlength spectrophotometry; solid-phase spectrophotometry.

\section{INTRODUÇÃO}

Espectrofotometria na região UV-VIS do espectro eletromagnético é uma das técnicas analíticas mais empregadas, em função de robustez, custo relativamente baixo e grande número de aplicações desenvolvidas ${ }^{1}$. Consultando-se o banco de dados do "Analytical Abstracts", verifica-se ca. 40000 ocorrências relacionadas à espectrofotometria ${ }^{2}$. Os procedimentos envolvem medidas diretas de espécies que absorvem radiação, medidas após derivação química e acoplamento a diversas técnicas ou processos, como cromatografia, eletroforese e análises em fluxo. Além disso, constitui-se em uma importante ferramenta para determinação de parâmetros fisico-químicos, tais como constantes de equilíbrio e de velocidade de reações.

Apesar da extensa utilização, várias inovações recentes relacionadas às medidas espectrofotométricas ainda são pouco conhecidas. Com respeito ao aspecto instrumental, é notável a evolução desde os procedimentos baseados em comparações visuais e dos fotômetros empregando filtros para a seleção de comprimentos de onda até os equipamentos mais recentes, que empregam arranjos de fotodetectores. Esses permitem a aquisição de espectros de absorção em intervalos de tempo da ordem de milésimos de segundos e são disponíveis, inclusive, em versões miniaturizadas acopladas a computadores do tipo "palm-top"3.

A espectrofotometria é fundamentada na lei de Lambert-Beer, que é a base matemática para medidas de absorção de radiação por amostras no estado sólido, líquido ou gasoso, nas regiões ultravioleta, visível e infravermelho do espectro eletromagnético. Para medidas de absorção de radiação em determinado comprimento de onda, temse: $\mathbf{A}=\log (\mathbf{I} / \mathbf{I})=\mathbf{\varepsilon b c}$, onde $\mathbf{A}$ é a absorvância, I é a intensidade da radiação monocromática que incide na amostra e $\mathbf{I}$ é a intensidade da radiação que emerge da amostra. A absortividade molar ( $\varepsilon$ ) é uma grandeza característica da espécie absorvente, cuja magnitude depende do comprimento de onda da radiação incidente. O termo c é a concentração da espécie absorvente e b, a distância percorrida pelo feixe através da amostra ${ }^{4}$.

Muitas das inovações referentes à espectrofotometria consistem em estratégias para aumento de sensibilidade, visando ampliação da

\footnotetext{
*e-mail: frprocha@iq.usp.br
}

faixa de aplicação da técnica e permitindo, em alguns casos, que medidas em concentrações da ordem de $n m o l ~ L^{-1}$ sejam efetuadas. Considerando-se a lei de Lambert-Beer, é possível reunir as alternativas para aumento de sensibilidade em três grupos. O primeiro refere-se ao emprego de estratégias para a formação de produtos com maiores absortividades molares, incluindo a síntese de novos reagentes e a formação de agregados micelares 5 . Em um segundo grupo, encontram-se estratégias para aumento do caminho óptico, permitindo que medidas sejam efetuadas com celas de até $5 \mathrm{~m}$ de caminho óptico ${ }^{6}$. O terceiro grupo refere-se a procedimentos que exploram a concentração da espécie a ser detectada previa ou simultaneamente à detecção ${ }^{7}$. Algumas das alternativas para aumento de sensibilidade em medidas espectrofotométricas são discutidas neste artigo.

\section{PRODUTOS COM MAIOR ABSORTIVIDADE MOLAR}

A constante de proporcionalidade que relaciona a absorvância com o caminho óptico e a concentração das espécies absorventes, denominada absortividade molar $(\varepsilon)$, empiricamente assume valores máximos da ordem de $10^{5} \mathrm{~L} \mathrm{~mol}^{-1} \mathrm{~cm}^{-1}$, para absorção de radiação na região UV-VIS. A magnitude de $\varepsilon$ depende da seção transversal de captura (S) da espécie e da probabilidade de ocorrência da transição eletrônica $(\mathrm{P})$, relacionada à absorção de radiação, da maneira descrita pela Equação $1^{8}$. Considerando-se esta equação, pode-se planejar modificações estruturais nos reagentes, de modo a maximizar a seção transversal de captura e/ou a probabilidade de ocorrência de transições eletrônicas, visando o aumento da absortividade molar da espécie.

$\varepsilon=8,7 \times 10^{19} \mathrm{PxS}$

Para a determinação espectrofotométrica de espécies na região UV-VIS, normalmente é necessário o uso de reagentes para a conversão da espécie de interesse em uma forma que permita a medida de absorção de radiação com maior sensibilidade e/ou seletividade. Sendo assim, espécies que absorvem fracamente podem ser convertidas em compostos com maior absortividade molar, visando a determinação de menores quantidades do analito ${ }^{9,10}$.

A sensibilidade e a seletividade de reagentes orgânicos usados para determinação espectrofotométrica podem ser modificadas pela 
incorporação de diferentes substituintes na molécula do reagente cromogênico. Por exemplo, dentre os vários reagentes disponíveis para determinações espectrofotométricas de metais, os compostos heterocíclicos contendo a estrutura $\alpha, \alpha$-diamina (-N=C-C=N-), conhecida como grupo funcional ferroína, são uma das classes de reagentes cromogênicos mais estudadas. Os compostos mais conhecidos desta classe são a 1,10-fenantrolina e a 2,2'-bipiridina, que formam quelatos estáveis e intensamente coloridos com ferro(II) e cobre(I). Várias modificações nas estruturas desses reagentes foram realizadas com o objetivo de melhorar a sensibilidade nas determinações espectrofotométricas e alguns exemplos são mostrados na Tabela 1. Da mesma forma, podem ser citados os reagentes da família da cuproína, conhecidos pela alta seletividade para a determinação de cobre(I). Exemplos de reagentes dessa família, que geram complexos de cobre com diferentes absortividades molares são 2,2'-biquinolina $\left(\varepsilon=6220 \mathrm{~L} \mathrm{~mol}^{-1} \mathrm{~cm}^{-1} \mathrm{em} 546 \mathrm{~nm}\right)$, neocuproína $\left(\varepsilon=7950 \mathrm{~L} \mathrm{~mol}^{-1} \mathrm{~cm}^{-1}\right.$ em $545 \mathrm{~nm}$ ) e batocuproína $\left(\varepsilon=13900 \mathrm{~L} \mathrm{~mol}^{-1} \mathrm{~cm}^{-1} \mathrm{em} 479 \mathrm{~nm}\right)^{11}$.

Outra estratégia que pode ser empregada para aumento da sensibilidade em determinações espectrofotométricas é a formação de complexos ternários. Reações entre um complexo $\mathbf{A B}$ e um terceiro componente $\mathbf{C}$ não necessariamente ocorrem pelo mecanismo de substituição regido pela diferença energética das ligações A-B e A-C (I). O terceiro componente pode vir a fazer parte do produto, formando um complexo ternário (II) ${ }^{12}$ :

$\mathrm{AB}+\mathrm{C} \leftrightarrows \mathrm{AC}+\mathrm{B}$

$\mathrm{AB}+\mathrm{C} \leftrightarrows \mathrm{ABC}$
Por exemplo, na interação do complexo azul de cobre(II)-piridina com ânions salicilato $\left(\mathrm{Sal}^{2-}\right)$ poderia se esperar a formação do composto verde, salicilato de cobre, de acordo com a reação:

$\left[\mathrm{Cu}^{2+} \cdot\right.$ piridina $\left._{2}\right]+\mathrm{Sal}^{2-} \leftrightarrows \mathrm{CuSal}+2$ piridina

Entretanto, em soluções contendo cobre(II), piridina e salicilato, um complexo azul é formado, com características espectrais diferentes dos compostos binários e com absortividade molar muito maior, devido à formação de um complexo ternário ${ }^{12}$. Da mesma maneira, ferro(III) forma um complexo amarelo com EDTA em ampla faixa de $\mathrm{pH}$, enquanto complexos estáveis com peróxido de hidrogênio não são usualmente observados. Entretanto, um complexo violeta é formado a partir da mistura de ferro(III), EDTA e $\mathrm{H}_{2} \mathrm{O}_{2}$ em pH 10.

Diferentes complexos ternários são formados com vanádio, resultando em aumento de sensibilidade para a determinação do íon metálico $^{13-15}$. Na reação de vanádio(IV) com o 2-(2-tiazolilazo)-pcresol (TAC), por exemplo, há a formação de um complexo verde com absorção máxima em 624 nm e absortividade molar de 2,36 x $10^{3} \mathrm{~L} \mathrm{~mol}^{-1} \mathrm{~cm}^{-1}$. Por outro lado, na presença de ácido ascórbico, o complexo formado possui coloração vermelha e absorção máxima em $525 \mathrm{~nm}$, com absortividade molar de $2,11 \times 10^{4} \mathrm{~L} \mathrm{~mol}^{-1} \mathrm{~cm}^{-1}{ }^{15}$.

A formação de complexos ternários pode ocorrer através de associação iônica, como ocorre com complexo catiônico formado entre um íon metálico e a 1,10-fenantrolina com o ânion vermelho de bromo-pirogalol ${ }^{16}$. Entretanto, sistemas micelares envolvendo surfactantes têm sido mais comumente usados ${ }^{17}$, visto que essas substâncias modificam algumas propriedades reacionais, podendo me-

Tabela 1. Efeito de alterações estruturais em ligantes sobre as absortividades molares de complexos de ferro e cobre ${ }^{5}$

\begin{tabular}{|c|c|c|c|c|}
\hline Ligante (L) & $\lambda_{\mathrm{MAX}} / \mathrm{nm}$ & $\begin{array}{l}\mathrm{L}_{2}^{+} \\
\varepsilon /\left(\mathrm{L} \mathrm{mol}^{-1} \mathrm{~cm}^{-1}\right)\end{array}$ & $\mathrm{FeL}_{3}^{2+}$ & ${ }^{3} \varepsilon /\left(\mathrm{L} \mathrm{mol}^{-1} \mathrm{~cm}^{-1}\right)$ \\
\hline 2,2'-bipiridina & 435 & 4500 & 522 & 8650 \\
\hline 1,10 -fenantrolina & 435 & 7000 & 510 & 11100 \\
\hline neocuproína ${ }^{\mathrm{a}}$ & 454 & 7950 & - & - \\
\hline batofenantrolina & 457 & 12140 & 533 & 22350 \\
\hline
\end{tabular}

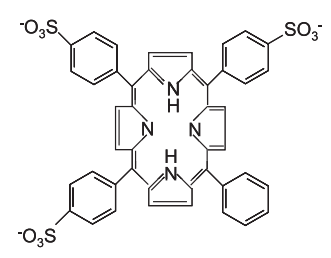

sulfonato de $\alpha, \beta, \gamma, \gamma$-tetrafenilporfirina ${ }^{\mathrm{b}}$

a. solvente: álcool isoamílico; b. complexos com estequiometria 1:1 
lhorar a sensibilidade e seletividade ${ }^{18}$. A presença de surfactantes pode ocasionar a formação de um novo complexo com diferentes propriedades espectrais (usualmente com maior absortividade molar), além de poder proporcionar também maior estabilidade e seletividade quando comparado com o complexo binário ${ }^{19}$. Estudos sobre os efeitos de surfactantes na formação de complexos permitem concluir que maiores modificações espectrais ocorrem quando a carga do surfactante responsável pela formação da micela é oposta à do íon do complexo formado entre o reagente cromogênico e o analito ${ }^{20}$. Por exemplo, o surfactante catiônico brometo de N-cetil-N,N,Ntrimetil amônio (CTAB) é usado na determinação espectrofotométrica de estanho com violeta de pirocatecol (PCV). Um complexo vermelho é formado entre o PCV e o estanho(IV) em pH 2,5, com absortividade molar estimada em $6,5 \times 10^{4} \mathrm{~L} \mathrm{~mol}^{-1} \mathrm{~cm}^{-1} \mathrm{em} 555 \mathrm{~nm}^{21}$. A presença de CTAB promove a formação de um complexo verde com absortividade molar de $1,0 \times 10^{5} \mathrm{~L} \mathrm{~mol}^{-1} \mathrm{~cm}^{-1} \mathrm{em} 660 \mathrm{~nm}^{22}$. Outro exemplo do uso do CTAB é a determinação de urânio com 2(2-tiazolilazo)-p-cresol (TAC). A presença de CTAB promove um aumento de sensibilidade, com absortividades molares estimadas em $1,31 \times 10^{4} \mathrm{~L} \mathrm{~mol}^{-1} \mathrm{~cm}^{-1}$ e $5,79 \times 10^{3} \mathrm{~L} \mathrm{~mol}^{-1} \mathrm{~cm}^{-1}$ na presença e na ausência do surfactante, respectivamente ${ }^{19}$. Outros exemplos do emprego de surfactantes em procedimentos espectrofotométricos com alta sensibilidade são apresentados na Tabela $2^{1,23-26}$.

\section{ESPECTROFOTOMETRIA DERIVATIVA}

A espectrofotometria derivativa consiste na representação das derivadas da absorvância em relação ao comprimento de onda, em função do comprimento de onda ${ }^{4}$. A diferenciação da lei LambertBeer permite obter as Equações 2-4, que mostram que as derivadas $\mathrm{d}^{\mathrm{n}} \mathrm{A} / \mathrm{d} \lambda^{\mathrm{n}}$ são sempre proporcionais às concentrações do analito, sendo as aplicações analíticas baseadas neste fato.

$\mathrm{dA} / \mathrm{d} \lambda=\mathrm{c} \mathrm{b}(\mathrm{d} \varepsilon / \mathrm{d} \lambda)$

$\mathrm{d}^{2} \mathrm{~A} / \mathrm{d} \lambda^{2}=\mathrm{c} \mathrm{b}\left(\mathrm{d}^{2} \varepsilon / \mathrm{d} \lambda^{2}\right)$

$\mathrm{d}^{\mathrm{n}} \mathrm{A} / \mathrm{d} \lambda^{\mathrm{n}}=\mathrm{c} b\left(\mathrm{~d}^{\mathrm{n}} \varepsilon / \mathrm{d} \lambda^{\mathrm{n}}\right)$

Os métodos de derivação espectral envolvem diferenciação eletrônica ou por software e são disponíveis em muitos espectrofotômetros $\mathrm{UV}-V I S^{27}$. Em geral, a derivação espectral visa determinações simultâneas, bem como aumento de seletividade. Entretanto, freqüentemente observa-se aumento de sensibilidade e melhoria do limite de detecção ${ }^{28,29}$. Um exemplo é a determinação de níquel com azul de hidroxinaftol (HNB) ${ }^{30}$. Níquel(II) reage com HNB em pH 5,7 formando um complexo vermelho estável, com máximo de absorção em 563 nm. O limite de detecção foi estimado em $23 \mu \mathrm{g} \mathrm{L} \mathrm{L}^{-1}$, quando se utilizou a espectrofotometria ordinária e $6 \mu \mathrm{g} \mathrm{L}^{-1}$ aplicando a primeira derivada. Aumento de sensibilidade foi também observado na determinação de níquel usando Br-PADAP como reagente cromogênico ${ }^{31}$. Limites de detecção de $1,8 \mu \mathrm{g} \mathrm{L}^{-1}$ e $0,2 \mu \mathrm{g} \mathrm{\textrm {L } ^ { - 1 }}$ foram estimados utilizando espectrofotometria ordinária e derivativa, respectivamente.
O aumento de sensibilidade observado na espectrometria derivativa é baseado na observação de que a amplitude da derivada $n$ da absorvância em relação ao comprimento de onda $\left(D_{n}=d^{n} \mathrm{~A} / \mathrm{d} \lambda\right)$ é inversamente proporcional à largura de banda do espectro ordinário $(\mathrm{W})^{28,32}$ :

$\mathrm{D}_{\mathrm{n}} \propto 1 / \mathrm{W}^{\mathrm{n}}$

Por esta razão, a sensibilidade em métodos derivativos depende não somente dos parâmetros instrumentais e da forma de medida do sinal (método da tangente, pico-a-pico ou zero-a-pico), como também das características do espectro de absorção ordinário ${ }^{28,29}$. A ordem da derivada deve ser cuidadosamente selecionada, visto que usualmente verifica-se um aumento do nível de ruído com o aumento da ordem de derivação ${ }^{33}$.

\section{AUMENTO DE CAMINHO ÓPTICO}

A sensibilidade de medidas espectrofotométricas também pode ser incrementada através do aumento do caminho óptico da cela de medida, devido ao conseqüente aumento no número de espécies absorventes que interagem com o feixe de radiação. Esse artifício é freqüentemente sugerido em procedimentos espectrofotométricos de rotina $^{34}$ sendo, entretanto, o caminho óptico limitado a ca. $5 \mathrm{~cm}$. Essa limitação surge devido à excessiva atenuação do feixe de radiação com o aumento do caminho óptico, devido à reflexão e refração pela solução e pelas paredes da cela.

O transporte de radiação por distâncias relativamente longas é eficientemente efetuado empregando-se cabos de fibras ópticas. Estes dispositivos consistem de um núcleo constituído por um material com índice de refração $\mathbf{n}_{1}$, recoberto por um material (revestimento) que apresenta menor índice de refração $\left(\mathbf{n}_{2}\right)$. O conjunto é usualmente recoberto por uma capa protetora, normalmente de plástico. Ambos os materiais devem ser transparentes, sendo usual o emprego de vidro ou polímeros. Se um feixe de radiação atinge o revestimento com ângulo $\theta_{1}$, parte da radiação é refletida dentro do cabo e parte é transmitida com ângulo de refração $\theta_{2}$. Os ângulos de incidência $\left(\theta_{1}\right)$ e de refração $\left(\theta_{2}\right)$ são relacionados através da lei de Snell (Equação 6). Como sen $\theta_{2}$ $\leq 1$, a equação 6 define um conjunto de ângulos de incidência (cone de aceitação), que implicarão na completa reflexão do feixe de radiação no interior do cabo de fibra óptica, com perdas desprezíveis por transmissão através do revestimento. Convém ressaltar que a capacidade da fibra óptica em coletar radiação externa (e portanto o cone de aceitação) depende do índice de refração do meio externo.

$\mathrm{n}_{1} \operatorname{sen} \theta_{1}=\mathrm{n}_{2} \operatorname{sen} \theta_{2} \Rightarrow \operatorname{sen} \theta_{2}=\mathrm{n}_{1} / \mathrm{n}_{2} \operatorname{sen} \theta_{1}$

O caminho óptico de medida pode ser aumentado empregandose celas que se comportem como guias de onda, de maneira similar ao que ocorre com os cabos de fibra óptica. Para tanto, o material do qual a cela é construída deve apresentar índice de refração inferior ao do líquido no qual a absorvância será medida ${ }^{35}$. Entretanto, os

Tabela 2. Exemplos de procedimentos baseados na formação de complexos ternários com surfactantes

\begin{tabular}{|c|c|c|c|c|c|}
\hline Analito & Reagente & Surfactante & $\lambda_{\mathrm{MAX}} / \mathrm{nm}$ & $\varepsilon /\left(\mathrm{L} \mathrm{mol}^{-1} \mathrm{~cm}^{-1}\right)$ & Ref \\
\hline $\mathrm{Ni}$ & Fenilfluorona & $\mathrm{CP}^{\mathrm{a}}$ & 540 & 100000 & 1 \\
\hline $\mathrm{Al}$ & Cromoazurol S & $\mathrm{HCP}^{\mathrm{b}}$ & 640 & 125000 & 23 \\
\hline $\mathrm{Co}$ & 5-Br-PADAP & Triton X-100 & 586 & 92400 & 24 \\
\hline $\mathrm{Fe}$ & Cromoazurol S & $\mathrm{CTA}^{\mathrm{c}}$ & 645 & 135000 & 25 \\
\hline V & Eriocromocianina $\mathrm{R}$ & $\mathrm{CTA}^{\mathrm{c}}$ & 575 & 79000 & 26 \\
\hline
\end{tabular}

a. CP - cloreto de cetilpiridinio; b. HCP- cloreto de hexadecilpiridinio e c. CTA- brometo de cetiltrimetilamônio. 
materiais usualmente empregados para a construção de celas espectrofotométricas apresentam índices de refração superiores ao da água, dificultando o uso para a construção de guias de onda para medidas em soluções aquosas.

A estratégia adotada inicialmente foi o aumento do índice de refração dos líquidos, através do emprego de solventes orgânicos ou adição de quantidades elevadas de solutos inertes ${ }^{35}$. Nesse sentido, foi proposto o emprego de celas constituídas por capilares de vidro com até $50 \mathrm{~m}$ de comprimento, preenchidas por solventes orgânicos com índice de refração superior ao do vidro $\left(\mathrm{n}_{\text {vidro }}=1,474\right)$. Estas celas permitiram um aumento de sensibilidade de 300 a 3000 vezes para a determinação de fosfato, iodeto, cobre e mercúrio em soluções de dissulfeto de carbono $(n=1,62)^{36}$. Apesar de engenhosas, essas alternativas são de pouca aplicação prática pois, usualmente, as medidas espectrofotométricas são efetuadas em soluções aquosas diluídas.

Outra alternativa explorada foi o emprego de celas capilares de aço inox $(1 \mathrm{~m})$ ou vidro revestido por fitas de alumínio (0,5-1 m), para favorecer a reflexão da radiação no interior do tubo e minimizar as perdas durante o transporte através da cela. Desta forma, foi possível aumentar 300 vezes a sensibilidade, alcançando limite de detecção de $5 \mathrm{ng} \mathrm{L}^{-1}$ para a determinação de fosfato pelo método do azul de molibdênio ${ }^{37}$.

O emprego de guias de onda para medidas em soluções aquosas diluídas (índice de refração em torno de 1,33) tornou-se mais usual com o desenvolvimento de um fluoropolímero amorfo, denominado Teflon AF- $2400^{\circledR}$, que apresenta índice de refração de $1,29^{38}$. Esses tubos podem ser empregados para construção de celas com longo caminho óptico, através de configurações similares à mostrada na Figura 1. Desta forma, foram desenvolvidos procedimentos espectrofotométricos para a determinação de diversas espécies, resultando em aumento de sensibilidade de até duas ordens de grandeza e limites de detecção da ordem de nmol L-1 (Tabela $3^{6,39-45}$ ).

$\mathrm{O}$ caminho óptico de medida também pode ser aumentado empregando-se celas de multi-reflexão. Embora essa estratégia seja bastante empregada para análise de gases, permitindo alcançar caminhos ópticos efetivos superiores a $100 \mathrm{~m}$ em medidas espectroscópicas no infravermelho ${ }^{46}$, o emprego para medidas em líquidos ainda é limitado. Alguns exemplos incluem a construção de celas para medidas por eletroforese capilar ${ }^{47}$ e análise por injeção em fluxo ${ }^{48}$, com aumento de sensibilidade de 40 e 1,7, respectivamente. Celas construídas com capilares de sílica fundida ${ }^{47}$ ou vidro borossilicato ${ }^{48}$ espelhados por um depósito de prata foram empregadas. Regiões dos capilares foram protegidas previamente ao depósito, visando obter janelas para entrada e saída do feixe de radiação proveniente de um laser de $\mathrm{He}-\mathrm{Ne}{ }^{47}$ ou de um diodo emissor de $\mathrm{luz}^{48}$. O número de reflexões internas foi estimado em $44^{47}$ e $19^{48}$ e o efeito do ângulo

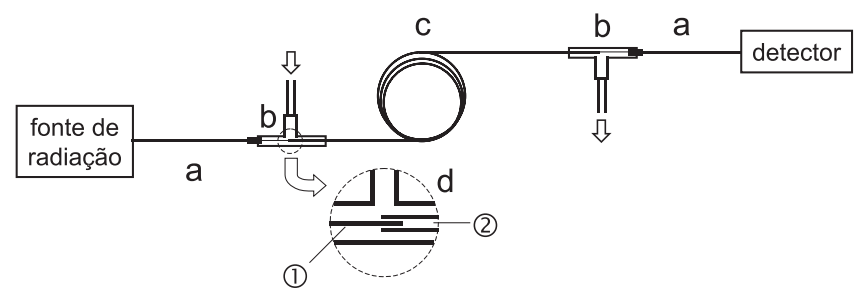

Figura 1. Arranjo experimental empregado para medidas espectrofotométricas com longo caminho óptico: a- cabos de fibra óptica; $b$ - adaptadores na forma de " $T$ " com volume interno reduzido empregados para alinhamento do feixe de radiação e para entrada e saída de soluções; c- guia de onda (tubo de Teflon AF-2400); d-vista ampliada de parte do adaptador para entrada de solução: o cabo de fibra óptica (1) é inserido no interior do guia de onda (2) para facilitar o alinhamento do feixe de radiação. A solução é introduzida pelo espaço entre o guia de onda e o cabo de fibra óptica em seu interior

de incidência da radiação foi avaliado e discutido.

\section{SEPARAÇÃO E CONCENTRAÇÃO DO ANALITO}

Devido às limitações de sensibilidade de metodologias com detecção espectrofotométrica, freqüentemente é necessário recorrer a etapas preliminares para separação e concentração dos elementos desejados, com consequiente aumento de sensibilidade. Dentre essas técnicas, pode-se citar a extração líquido-líquido, precipitação e extração sólido-líquido.

Na extração líquido-líquido, um solvente no qual a espécie de interesse apresente maior solubilidade é empregado para favorecer a transferência de fase da solução da amostra para o solvente. Conseqüentemente, ocorre a separação e, como o solvente é usualmente empregado em quantidades menores que a amostra, a concentração do analito. No caso da determinação de íons metálicos, muitos reagentes orgânicos podem ser usados para a formação de complexos, possibilitando a transferência de fase. O 1-(2-piridilazo)-2-naftol (PAN), por exemplo, pode ser utilizado para extração de $\mathrm{Fe}(\mathrm{III}), \mathrm{Cr}$ (III), $\mathrm{Mn}$ (II), Ni(II), Cu(II) e $\mathrm{Zn}(\mathrm{II})$, entre outros, através de seus quelatos poucos solúveis em meio aquoso, empregando solventes orgânicos apropriados, para posterior quantificação por espectrofotometria UV-visível ${ }^{49}$. Devido à baixa solubilidade de vários quelantes, estes reagentes também podem ser usados em procedimentos de separação e pré-concentração por precipitação. Por exemplo, quando uma solução alcoólica de PAN é adicionada a uma amostra aquosa, o reagente se cristaliza e precipita vários complexos metálicos por co-cristalização ${ }^{50}$. Esses complexos podem

Tabela 3. Procedimentos com alta sensibilidade empregando espectrofotometria com longo caminho óptico

\begin{tabular}{|c|c|c|c|c|c|}
\hline Espécie & $\begin{array}{l}\text { Caminho óptico } \\
(\mathrm{cm})\end{array}$ & $\begin{array}{l}\text { Faixa de resposta } \\
\left.\qquad(\mathrm{nmol} \mathrm{L})^{-1}\right)\end{array}$ & $\begin{array}{l}\text { Sensibilidade } \\
\left(\mathrm{L} \mu \mathrm{mol}^{-1}\right)\end{array}$ & $\begin{array}{l}\text { Limite de detecção } \\
\quad\left(n m o l ~ L^{-1}\right)\end{array}$ & Ref \\
\hline $\mathrm{Cr}(\mathrm{VI})$ & 500 & $1-30$ & $22,3 \pm 0,1$ & 0,2 & 6 \\
\hline $\mathrm{Mo}(\mathrm{VI})$ & 500 & $2-30$ & $27,0 \pm 0,1$ & 0,6 & 6 \\
\hline $\mathrm{Fe}(\mathrm{II})$ & 447 & $0,5-10$ & $10,6 \pm 0,2$ & 0,2 & 39 \\
\hline $\mathrm{Fe}(\mathrm{II})$ & 200 & $1-50$ & 4,11 & 0,1 & 40 \\
\hline $\mathrm{NO}_{2}^{-}$ & 450 & $<30$ & $20,4 \pm 0,3$ & 0,5 & 41 \\
\hline $\mathrm{NO}_{3}^{-}$ & 450 & $<30$ & $18,4 \pm 0,8$ & 1,5 & 41 \\
\hline $\mathrm{H}_{2} \mathrm{~S}^{3}$ & 160 & - & $2,40 \pm 0,08$ & 5 & 42 \\
\hline $\mathrm{Cu}^{2}(\mathrm{II})$ & 440 & $1-160$ & $6,13 \pm 0,02$ & 0,4 & 43 \\
\hline Fenóis & 100 & $100-1000$ & - & 10 & 44 \\
\hline $\mathrm{Cr}(\mathrm{VI})$ & 50 & $<900$ & - & 100 & 45 \\
\hline $\mathrm{Al}(\mathrm{III})$ & 50 & $<30000$ & - & 180 & 45 \\
\hline
\end{tabular}


ser posteriormente solubilizados para determinação por espectrofotometria UV-visível.

Entre os procedimentos de separação e concentração para a determinação por espectrofotometria, a extração em fase sólida tem sido a mais utilizada, devido à simplicidade e aos melhores fatores de enriquecimento do analito ${ }^{51}$. A separação é baseada na sorção do analito na fase sólida, que pode estar associada a um reagente orgânico, e à eluição com solvente apropriado. Elevados fatores de concentração podem ser alcançados pelo emprego de um grande volume de amostra e eluição com um pequeno volume de solvente.

\section{MEDIDAS EM FASE SÓLIDA}

Medidas de absorvância podem realizadas diretamente em um suporte sólido, no qual o analito foi retido. A primeira proposta neste sentido foi feita por Yoshimura e colaboradores ${ }^{52}$, que empregaram uma resina de troca iônica com difenilcarbazida, 1,10fenantrolina, tiocianato de amônio ou Zincon imobilizados na fase sólida para a determinação de cromo, ferro, cobalto e cobre, respectivamente. Desde então, o uso da espectrofotometria em fase sólida (EFS) vem crescendo devido à simplicidade, facilidade de automação e baixos limites de detecção que podem ser alcançados ${ }^{7,53}$. Na EFS, uma matriz sólida é utilizada para retenção da espécie de interesse, permitindo a concentração do analito, que é acumulado em um pequeno volume da fase sólida ${ }^{54-57}$. Além do aumento de sensibilidade, outras vantagens podem ser observadas, como a integração de etapas de reação, retenção e detecção; menor consumo de reagentes e aumento de seletividade.

A retenção do analito na fase sólida pode ocorrer devido à interação direta da espécie com a fase sólida ${ }^{58,59}$, à retenção de um produto da reação entre o analito e um reagente ${ }^{60-62}$, ou à retenção do analito por um reagente previamente imobilizado no suporte sólido $^{63,64}$. A aplicação de uma destas estratégias dependerá da espécie química a ser analisada, do suporte sólido e da reação envolvida.

Assim como os procedimentos de pré-concentração em fase sólida, a EFS pode ser implementada em batelada ou em fluxo. Os procedimentos em batelada envolvem o contato direto do analito com a fase sólida em um recipiente sob agitação, para posterior transferência para a cela de medida. As medidas são geralmente efetuadas em mais de um comprimento de onda, visando compensar diferenças de empacotamento da fase sólida. Nos procedimentos em fluxo, utiliza-se uma coluna ou uma cela que possa ser adaptada diretamente no caminho óptico de um espectrofotômetro, sendo as medidas efetuadas simultaneamente à retenção da espécie de interesse. Desta forma, o tempo de análise é consideravelmente reduzido e as diferenças de empacotamento da fase sólida entre as medidas são evitadas $^{65,66}$.

Alguns pré-requisitos desejáveis para emprego de um sistema para medidas por espectrofotometria em fase sólida são ${ }^{63,67}$ : reversibilidade, estabilidade da fase sólida no meio de trabalho, transparência do suporte sólido e cinética de retenção rápida. O último fator é particularmente importante em sistemas em fluxo, que usualmente trabalham com tempos de residência curtos. Neste caso, a retenção rápida do analito permite fatores de extração maiores e, conseqüentemente, maior sensibilidade, sem comprometer a freqüência de amostragem.

A sensibilidade das medidas por EFS depende do caminho óptico ocupado pela fase sólida e do volume de amostra utilizado na determinação. Alguns exemplos do aumento de sensibilidade em relação à espectrofotometria convencional e dos limites de detecção que podem ser alcançados são apresentados na Tabela $4^{68-72}$.

Frenzel e Krekler ${ }^{60}$ compararam três estratégias para a determinação espectrofotométrica de fenóis. A primeira envolveu pré-concentração do produto da reação entre fenol e 4-aminoantipirina (4-AAP) por extração com $\mathrm{CCl}_{4}$. A segunda foi baseada na extração/concentração do produto utilizando sílica funcionalizada $\mathrm{C}_{18}$, eluição com metanol e posterior determinação. No terceiro procedimento, a absorvância foi monitorada diretamente na fase sólida. Os limites de detecção encontrados foram 8, 11 e $0,4 \mu \mathrm{g} \mathrm{L}^{-1}$, respectivamente. Este exemplo ilustra o aumento de sensibilidade quando se utiliza EFS em comparação com procedimentos para pré-concentração em fase sólida, nos quais o analito deve ser eluído previamente à medida, sendo inerente a perda de sensibilidade por diluição.

\section{OUTRAS ALTERNATIVAS}

Os procedimentos descritos anteriormente referem-se à medida da quantidade de radiação absorvida pela amostra, denominados métodos de transmissão ou espectrofotometria convencional. Apesar desta estratégia ser a mais amplamente utilizada, existem métodos alternativos baseados na medida da potência absorvida pela amostra, denominados métodos calorimétricos. Esses procedimentos são inerentemente mais sensíveis que a espectrofotometria convencional, apresentando limites de detecção da ordem de $10^{-7} \mathrm{~mol} \mathrm{~L}^{-1}$, de duas a três ordens de magnitude inferiores aos obtidos por medidas diretas de absorção ${ }^{1,73}$. As duas principais técnicas deste grupo são a espectrofotometria de lentes térmicas e a espectrofotometria fotoacústica. Na espectrofotometria de lentes térmicas, são medidas alterações de índice de refração da solução do analito, em função da variação de temperatura que ocorre devido à absorção de radiação.

Tabela 4. Exemplos de procedimentos com alta sensibilidade explorando espectrofotometria em fase sólida

\begin{tabular}{|c|c|c|c|c|}
\hline Espécie & Reagente/suporte & Limite de detecção $\left(\mu \mathrm{g} \mathrm{L}^{-1}\right)$ & $\mathrm{V}_{\text {amostra }}(\mathrm{mL})$ & Ref \\
\hline $\mathrm{Zn}(\mathrm{II})$ & 1-(2-tiazolylazo)-2-naftol / sílica funcionalizada $\mathrm{C}_{18}$ & 10 & 0,6 & 56 \\
\hline $\mathrm{S}^{2-}$ & $\mathrm{N}, \mathrm{N}$-dimetil-p-fenilenediamina/ $\mathrm{Fe}^{3+} /$ sílica funcionalizada $\mathrm{C}_{18}$ & $1,7^{\mathrm{a}}$ & 0,5 & 57 \\
\hline $\mathrm{Cu}(\mathrm{II})$ & 1-(2-piridilazo)-2-naftol / resina aniônica (Dowex 50W-X4) & 1 & 0,9 & 63 \\
\hline $\mathrm{Fe}(\mathrm{III})$ & $\mathrm{SCN}^{-} /$resina aniônica (Dowex 1-X2) & 10 & 2 & 64 \\
\hline $\mathrm{V}(\mathrm{IV})$ & Alaranjado de xilenol / resina aniônica AG 1-X2 & $0,8^{\mathrm{b}}$ & 5 & 68 \\
\hline $\mathrm{Cr}(\mathrm{VI})$ & 1,5-difenilcarbazida / resina catiônica AG 50W-X2 & $0,1^{\mathrm{c}}$ & 4,4 & 69 \\
\hline $\mathrm{PO}_{4}^{3-}$ & Molibdato de amônio; verde de malaquita / Sephadex LH-20 & 0,2 & 4,4 & 70 \\
\hline sulfatiazol & Retenção direta / Sephadex SP C-25 gel & 20 & 250 & 71 \\
\hline \multirow[t]{2}{*}{$\mathrm{V}(\mathrm{IV})$} & Eriocromo cianinaR / Sephadex DEAE A-25 & 3 & 250 & 72 \\
\hline & & 0,6 & 1000 & \\
\hline
\end{tabular}

a. Limite de detecção 30 vezes inferior ao obtido em procedimento em solução empregando mesmo volume de amostra; b. sensibilidade 280 vezes superior à obtida por medidas em solução e c. sensibilidade 160 vezes superior à obtida por medidas em solução. 
Além da elevada sensibilidade, é possível efetuar medidas com pequenos volumes de amostra e a técnica apresenta faixa dinâmica de resposta consideravelmente maior que a espectrofotometria convencional $^{1,74}$. A espectrofotometria fotoacústica (ou optoacústica) explora a expansão térmica da amostra irradiada e pode ser aplicada a gases, sólidos e líquidos. Descrições detalhadas dos conceitos, instrumentação e aplicações destas técnicas podem ser encontradas em artigos de revisão ${ }^{73-75}$.

\section{CONCLUSÕES E PERSPECTIVAS}

As estratégias descritas neste artigo permitem aumentar consideravelmente a sensibilidade de procedimentos espectrofotométricos e, conseqüentemente, ampliar a aplicação da técnica a análise de traços. Desta forma, os procedimentos espectrofotométricos podem ser revisitados e características favoráveis como robustez, baixo custo e simplicidade, podem torná-los competitivos com outras metodologias dedicadas à determinação de espécies químicas em baixas concentrações. Algumas das estratégias descritas, como o aumento do caminho óptico e as medidas diretas em fase sólida, são de aplicação geral e a disseminação de seu uso depende, principalmente, da disponibilidade comercial de instrumentação adequada. Celas construídas a partir de guias de onda são comercialmente disponíveis. Estas celas apresentam caminho óptico de até $500 \mathrm{~cm}$ e volumes relativamente reduzidos (ca. $2,5 \mu \mathrm{L} / \mathrm{cm}$ ), sendo adequadas para medidas espectrofotométricas inclusive em sistemas de análises em fluxo. As medidas em fase sólida, por outro lado, têm sido implementadas empregando espectrofotômetros dedicados a medidas em solução, o que resulta em limitações da quantidade de suporte sólido que pode ser empregada. Apesar dessas limitações poderem ser superadas em laboratórios de pesquisa, por exemplo pela construção de celas especiais ou pelo emprego de fontes de radiação com maior potência, a disponibilidade de equipamentos dedicados é certamente importante para ampliar a sua utilização.

\section{AGRADECIMENTOS}

Os autores agradecem ao CNPq, Finep e FAPESP pelas bolsas e apoio financeiro.

\section{REFERÊNCIAS}

1. Lobinski, R.; Marczenko, Z.; Crit. Rev. Anal. Chem. 1992, 23, 55.

2. Banco de dados Analytical Abstracts, http://www.portaldapesquisa.com.br/ databases/sites, acessada em Maio 2003.

3. http://www.oceanoptics.com, acessada em Março 2003.

4. Perkampus, H. H.; UV-VIS Spectroscopy and its Applications, SpringerVerlag: Berlin, 1992.

5. Cheng, K. L.; Ueno, K.; Imamura, T.; Handbook of Organic Analytical Reagents, CRC Press: Boca Raton, 1982.

6. Yao, W.; Byrne, R. H.; Talanta 1999, 48, 277.

7. Fang, Z.; Flow Injection Separation and Preconcentration, VCH: Weinheim, 1993.

8. Braude, E. A.; J. Chem. Soc. 1950, 379.

9. Howell, J. A.; Hargis, L. G.; Anal. Chem. 1994, 66, 445R.

10. Prenesti, E.; Daniele, P. G.; Toso, S.; Anal. Chim. Acta 2002, 459, 323.

11. Stephen, W. I.; Islam, M. A.; Anal. Chim. Acta 1993, 274, 347.

12. Babko, A. K.; Talanta 1968, 15, 721.

13. Lukachina, V.; Pilipenko, A. T.; Karpova, O. I.; Zh. Anal. Khim. 1973, 28, 70.

14. Kiss, E.; Anal. Chim. Acta 1975, 77, 205

15. Teixeira, L. S. G.; Costa, A. C. S.; Queiroz, A. S.; Amorim, A. M. M.; Bonfim, O. N.; Ferreira, S. L. C.; Mikrochim. Acta 1998, 129, 103.

16. Bailey, B. W.; Chester, J. E.; Dagnall, R. M.; West, T. S.; Talanta 1968, $15,1359$.

17. Diaz-Garcia, M. E.; Sanz-Mendel, A.; Talanta 1985, 32, 189.

18. Maniasso, N.; Quim. Nova 2001, 24, 87.

19. Teixeira, L. S. G.; Costa, A. C. S.; Ferreira, S. L. C.; Freitas, M. L.; Carvalho, M. S.; J. Braz. Chem. Soc. 1999, 10, 519.
20. Svobova, V.; Chromy, V.; Talanta 1965, 12, 431.

21. Ross, W. J.; White, J. C.; Anal. Chem. 1961, 33, 421.

22. Costa, A. C. S; Teixeira, L. S. G.; Ferreira, S. L. C; Talanta 1995, 42, 1973.

23. Sampson, B.; Fleck, A.; Analyst 1984, 109, 369.

24. Zbiral, J.; Sommer, L.; Fresenius J. Anal. Chem. 1981, 306, 129.

25. Marczenko, Z.; Kalowska, H.; Anal. Chim. Acta 1981, 123, 279.

26. Jarosz, M.; Marczenko, Z.; Analyst 1984, 109, 35.

27. Howell, J. A.; Hargis, L. G.; Anal. Chem. 1990, 62, 155R.

28. Medilla, J.; Ales, F.; Sanchez, F. G.; Talanta 1986, 33, 329.

29. Sedaira, H.; Talanta 2000, 51, 39.

30. Ferreira, S. L. C.; Santos, B. F.; Andrade, J. B.; Costa, A. C. S.; Mikrochim. Acta 1996, 122, 109.

31. Ferreira, S. L. C.; Costa, A. C. S.; de Jesus, D. S.; Talanta 1996, 43, 1649.

32. Rojas, F. S.; Ojeda, C. B.; Pavon, J. M. C.; Talanta 1988, 35, 753.

33. O'Haver, T. C.; Begley, T.; Anal. Chem. 1981, 53, 1876.

34. Eaton, A. D.; Clesceri, L. S.; Greenberg, A. E.; Standard Methods for the Examination of Water and Wastewater, $19^{\text {th }}$ ed., American Public Association: Washington, 1995.

35. Dasgupta, P. K.; Genfa, Z.; Poruthoor, S. K.; Caldwell, S.; Dong, S.; Liu, S.; Anal. Chem. 1998, 70, 4661.

36. Fuwa, K.; Lei, W.; Fujiwara, K.; Anal. Chem. 1984, 56, 1640.

37. Lei, W.; Fujiwara, K.; Fuwa, K.; Anal. Chem. 1983, 55, 951.

38. http://www.biogeneral.com/teflonaf.htm, acessada em Janeiro 2003.

39. Waterbury, R. D.; Yao, W.; Byrne, R. H.; Anal. Chim. Acta 1997, 357, 99

40. Zhang, J. Z.; Kelble, C.; Millero, F. J.; Anal. Chim. Acta 2001, 438, 49

41. Yao, W.; Byrne, R. H.; Waterbury, R. D.; Environ. Sci. Technol. 1998, 32, 2646.

42. Byrne, R. H.; Yao, W.; Kaltenbacher, E.; Waterbury, R. D.; Talanta 2000, 50, 1307.

43. Callahan, M. R.; Rose, J. B.; Byrne, R. H.; Talanta 2002, 58, 891.

44. Lupetti, K. O.; Rocha, F. R. P.; Fatibello-Filho, O.; Talanta 2004, 62, 463.

45. Li, Q.; Morris, K. J.; Dasgupta, P. K.; Raimundo, I. M.; Temkin, H.; Anal. Chim. Acta 2003, 479, 151.

46. Willard, H. H.; Merritt, L. L.; Dean, J. A.; Settle, F. A.; Instrumental Methods of Analysis, $7^{\text {th }}$ ed., Wadsworth: Belmont, 1988, p. 306.

47. Wang, T.; Aiken, J. H.; Huie, C. W.; Hartwick, R. A.; Anal. Chem. 1991, 63, 1372.

48. Ellis, P. S.; Lyddy-Meaney, A. J.; Worsfold, P. J.; McKelvie, I. D.; Anal. Chim. Acta 2003, 499, 81

49. Cheng, K. L.; Bray, R. H.; Anal. Chem. 1955, 27, 782.

50. Vanderstappen, M. G.; van Grieken R. E.; Talanta 1978, 25, 653.

51. Ruzicka, J.; Arndal, A.; Anal. Chim. Acta 1989, 216, 243.

52. Yoshimura, K.; Waki, H.; Ohashi, S.; Talanta 1976, 23, 449.

53. Valcárcel, M.; Luque de Castro, M. D.; Analyst 1990, 115, 699.

54. Pascual-Reguera, M. I.; Molina-Diaz, A.; Ramos-Martos, N.; Anal. Lett. 1991, 24, 2245

55. Teixeira, L. S. G.; Rocha, F. R. P.; Korn, M.; Reis, B. F.; Ferreira, S. L. C.; Costa, A. C. S.; Talanta 2000, 51, 1027.

56. Teixeira, L. S. G.; Rocha, F. R. P.; Korn. M.; Reis, B. F.; Ferreira, S. L. C.; Costa, A. C. S.; Anal. Chim. Acta 1999, 383, 307.

57. Cassella, R. J.; Teixeira, L. S. G.; Garrigues, S.; Costa, A. C. S.; de la Guardia, M.; Analyst 2000, 125, 1835.

58. Yoshimura, K.; Anal. Chem. 1987, 59, 2922.

59. Fernández-Band, B.; Lázaro, F.; Luque de Castro, M. D.; Valcárcel, M.; Anal. Chim. Acta 1990, 229, 177.

60. Frenzel, W.; Krekler, S.; Anal. Chim. Acta 1995, 310, 437.

61. Linares, P.; Luque de Castro, M. D.; Valcárcel, M.; Anal. Chim. Acta 1990, $230,199$.

62. Capitan-Vallvey, L. F.; Valencia, M. C.; Mirón, G.; Anal. Chim. Acta 1994, $289,365$.

63. Lazaro, F.; Luque de Castro, M. D.; Valcárcel, M.; Anal. Chim. Acta 1988, $214,217$.

64. Lazaro, F.; Luque de Castro, M. D.; Valcárcel, M.; Anal. Chim. Acta 1989, 219,231

65. Reis, B. F.; Rocha, F. R. P.; Teixeira, L. S. G.; Costa, A. C. S.; Korn, M.; Quim. Nova 2000, 23, 116.

66. Teixeira, L. S. G.; Costa, A. C. S.; Assis, J. C. R.; Ferreira, S. L. C.; Korn M.; Mikrochim. Acta 2001, 137, 29.

67. Vereda, E.; Rios, A.; Valcarcel, M.; Analyst 1997, 122, 85.

68. Matsuoka, S.; Yoshimura, K.; Tateda, A.; Anal. Chim. Acta 1995, 317, 207.

69. Yoshimura, K.; Analyst 1988, 113, 471.

70. Yoshimura, K.; Nawata, S.; Kura, G.; Analyst 1990, 115, 843.

71. Capitan-Vallvey, L. F.; Orbe, I.; Valencia, M. C.; Berzas-Nevado, J. J.; Talanta 1994, 41, 1327.

72. Boudra, S.; Bosque-Sendra, J. M.; Valencia, M. C.; Talanta 1995, 42, 1525.

73. Harris, T. D.; Anal. Chem. 1982, 54, 741A

74. Ramos, G. R.; Anal. Chim. Acta 1993, 283, 623.

75. Betteridge, D.; Meylor, P. J.; Crit. Rev. Anal. Chem. 1984, 14, 267. 